\title{
Background radiation in direct dark matter experiments
}

\author{
Vitaly A. Kudryavtsev* \\ Department of Physics and Astronomy, University of Sheffield, Sheffield, S3 7RH, UK \\ E-mail: v.kudryavtsev@sheffield.ac.uk
}

\begin{abstract}
Gamma-rays and neutrons are the most important backgrounds in high-sensitivity experiments for direct dark matter searches. They are produced in the decays of the radioactive isotopes in rock and detector materials. Neutrons are originated in the spontaneous fission and $(\alpha, n)$ reactions from the decays of uranium and thorium and their daughters. The flux of gammarays and neutrons can be attenuated and suppressed by passive and active shielding (including self-shielding). Cosmic-ray muons are responsible for producing high-energy neutrons that can travel from large distances avoiding active veto systems, hitting the target and giving a signal similar to that from WIMPs. Gamma-rays can be discriminated from the WIMP-like interactions using different methods. This paper briefly discusses the status of background studies for direct WIMP dark matter searches in connection with a proposed tonne-scale cryogenic experiment with multiple targets EURECA. Major sources of background, such as radioactivity and cosmic rays, are considered.
\end{abstract}

Identification of Dark Matter 2010-IDM2010

July 26-30, 2010

Montpellier France

${ }^{*}$ Speaker. 


\section{Introduction}

The sensitivity of large-scale underground detectors to direct WIMP searches, neutrinoless double-beta decay, and low-energy neutrino studies are limited by various types of background radiation. Knowledge of gamma-ray and neutron fluxes and ability to suppress or reject background events are essential for estimating detector sensitivity, interpreting experimental results and designing future experiments.

In this paper we present a short overview of neutron and gamma-ray background, and methods of suppressing background events caused by different sources. The results reported here, have been obtained mainly by calculating the background for dark matter experiments but may also be useful for modelling the radioactive background for other underground detectors. Neutrons underground arise from two sources: i) local radioactivity, and ii) cosmic-ray muons. Neutrons associated with local radioactivity are produced via spontaneous fission of ${ }^{238} \mathrm{U}$ and $(\alpha, \mathrm{n})$ reactions initiated by $\alpha$-particles from U/Th traces in rock and detector components. The muon-induced neutron flux depends strongly on the muon flux, i.e. the depth of the laboratory site. Gamma-ray background originated in rock and detector components exceeds the neutron one by several orders of magnitude even beyond the shielding. There are, however, powerful techniques that allows discrimination of this type of background in dark matter experiments.

\section{Gamma-ray and neutron production}

The gamma-ray production in the decay chains of uranium (U) and thorium (Th), and in the decay of other unstable isotopes $\left({ }^{40} \mathrm{~K},{ }^{60} \mathrm{Co}\right.$ etc. $)$ can be calculated using the well-known codes and toolkits, such as GEANT4 [1] and DECAY0 [2]. The comparison between the two codes shows a good agreement except for the energy range below $100 \mathrm{keV}$ where DECAY0 does not claim high accuracy [2] whereas GEANT4 misses some Auger electrons.

The production of neutrons in spontaneous fission and $(\alpha, n)$ reactions is not implemented in GEANT4 and has to be calculated using different codes. A well established way is to use SOURCES4 [3]. The code is well tested and was used extensively in nuclear physics calculations. The original code was limited to $\alpha$-energies below $6.5 \mathrm{MeV}$. The code has been modified [4] to extend the range of alpha energies to $10 \mathrm{MeV}$. The measured cross-sections of $(\alpha, n)$ reactions on several isotopes above $6.5 \mathrm{MeV}$ have been added to the code library. Further improvements to the code [5, 6] included theoretical cross-sections calculated with EMPIRE2.19 [7].

The comparison of EMPIRE2.19 cross-sections and SOURCES4A spectra and yields with experimental data can be found in $[6,8]$ and in the original user manual for SOURCES4. A good agreement has been found between EMPIRE2.19 calculations and data for isotopes where data are available, proving the validity of EMPIRE2.19 code.

Neutron spectra at production depend strongly on the material. Materials containing isotopes with low energy thresholds for $(\alpha, n)$ reactions, such as $\mathrm{NaCl}$, may give a relatively high neutron flux compared to other types of materials or rocks, for a fixed concentrations of U/Th. Rocks with high content of water (for instance, Modane and Gran Sasso rocks) have an advantage compared to other rocks since hydrogen is a good neutron moderator and the high-energy neutron flux on the rock/lab interface is attenuated by neutron moderation in rock more than in the absence of water. 


\section{Particle transport and shielding calculations}

The modelling of transport of gamma-rays and neutrons through large thickness of materials is important for designing shielding for underground particle astrophysics experiments. The transport of gamma-rays and neutrons is usually carried out using GEANT4 [1]. There are two typical shielding arrangements realised in the current, and considered for future experiments: (i) high- $Z$ and low-A materials arranged in the interleaved layers (minimum one layer of each is required to attenuate both gamma-ray and neutron fluxes); (ii) large amount of water that protects the detector from both neutrons and gamma-rays. In the later case water can also be instrumented with PMTs playing a role of an active veto system against muons and muon-induced events. There are obvious variations from the two main schemes, such as using external part of the target volume as a shield and active veto system (xenon and argon based experiments), using liquid scintillator (sometimes loaded with $\mathrm{Gd}$ ) around the target as an active veto system against neutrons and gamma-rays produced in the detector components etc (see presentations at IDM2010 on this subject).

Simulations reported in Refs. [4, 5, 8, 9] and other papers showed that about 20-25 cm of lead and $60 \mathrm{~cm}$ of polyethylene would attenuate the gamma-ray and neutron fluxes from the cavern walls by about 6 orders of magnitudes guaranteeing the background event rate after discrimination in the region of interest for dark matter experiments below one event per tonne of target per year. Water, being a good moderator of neutrons, is much less efficient in attenuating high-energy gamma-ray flux due to its low density. At least 3 metres of water is needed to attenuate gamma-ray flux to a level that would guarantee the required sensitivity of the tonne-scale dark matter experiments $[8,9]$. Exact thickness depends on the target material and discrimination power. Figures 1 and 2 show the energy spectra of gamma-rays and electrons behind different thicknesses of water as simulated with GEANT4 [10]. The gamma-rays were produced by allowing the radioisotopes of $U$ and Th and their daughters to decay in GEANT4. U and Th were assumed to have concentrations of $1.9 \mathrm{ppm}$ and $1.4 \mathrm{ppm}$ in concrete as measured in the concrete that covers the walls of the Modane underground laboratory.

Water as a shield can be around the cryostat in a water tank (detector immersed in water - swimming pool solution) or around the whole lab along the walls (submarine solution). The advantage of the submarine solution is the easy access to the detector without the need to remove detector from the water. It appears, however, that in the submarine solution the detector is exposed to the additional background produced in the walls of the tanks containing water. Assuming the water container(s) is made of $2 \mathrm{~cm}$ thick stainless steel with a typical U/Th concentrations of 1 ppb, an event rate of about 15-20 nuclear recoils and up to $10^{6}$ electron recoils per year at 10-50 $\mathrm{keV}$ is expected in $100 \mathrm{~kg}$ of Ge. To attenuate this background, an additional shielding around the detector is required, eliminating the advantage of the easy access to the detector.

When the background from the walls is sufficiently attenuated, the radiation from detector components may become the limiting factor for high-sensitivity experiments. The studies of the radiation from detector components are on-going for several existing and future projects. Initial results for a planned large-scale cryogenic dark matter detector EURECA have been reported in Refs. $[8,10]$. A large-mass of $\mathrm{Cu}$ used in cryostat, support plates, crystal holders and caps, need to be very radio-pure to keep background event rate to the minimum. Figure 3 shows the electron recoil spectra in $506 \mathrm{~kg}$ of Ge from $0.01 \mathrm{ppb} \mathrm{U}, 0.01 \mathrm{ppb}$ Th and $10 \mathrm{ppb}$ of natural potassium $(\mathrm{K})$ 


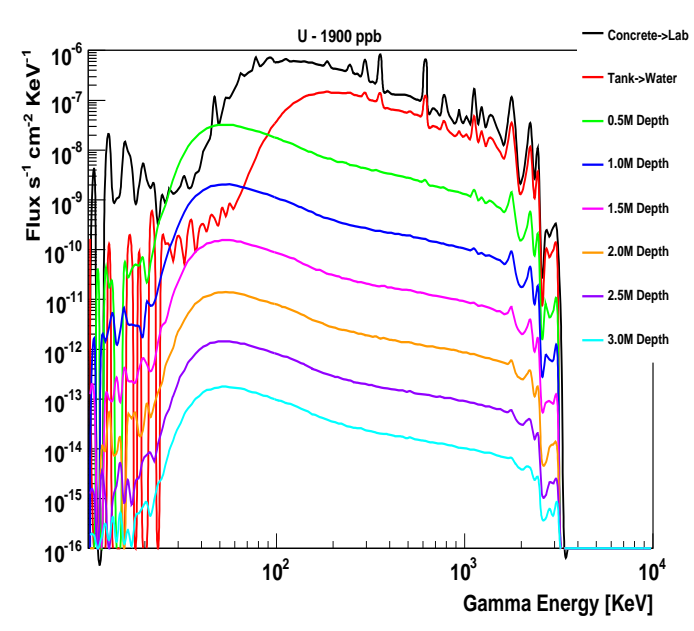

Figure 1: Energy spectra of gamma-rays and electrons from $U$ decay chain in equilibrium, behind different thicknesses of water [10].

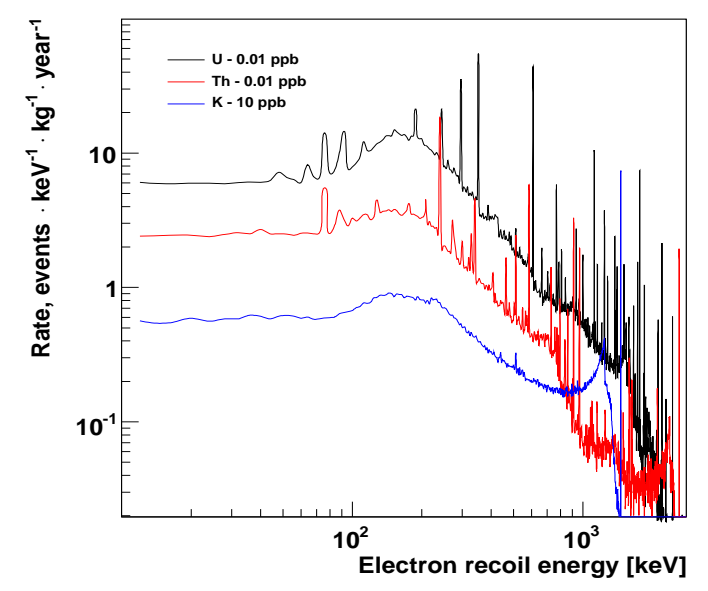

Figure 3: Electron recoil spectra in $506 \mathrm{~kg}$ of Ge from $0.01 \mathrm{ppb} \mathrm{U}, 0.01 \mathrm{ppb}$ Th and $10 \mathrm{ppb}$ of natural potassium $(\mathrm{K})$ in a cryostat and internal parts made out of 2.9 tonnes of copper [10].

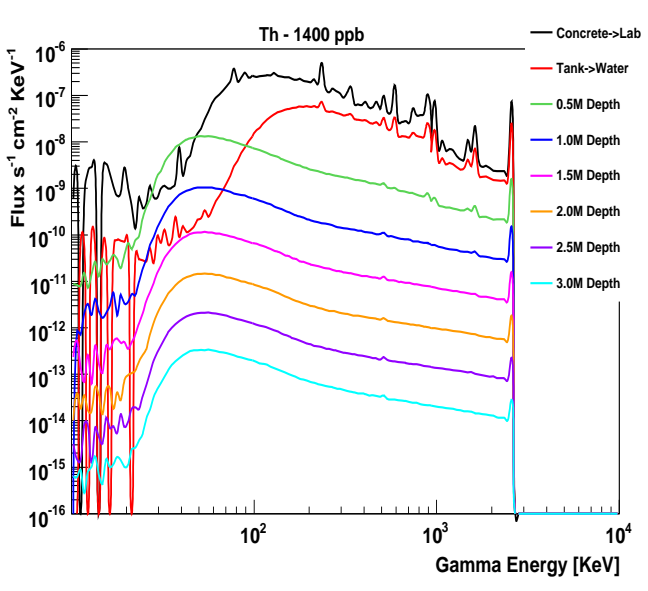

Figure 2: Energy spectra of gamma-rays and electrons from Th decay chain in equilibrium, behind different thicknesses of water [10].

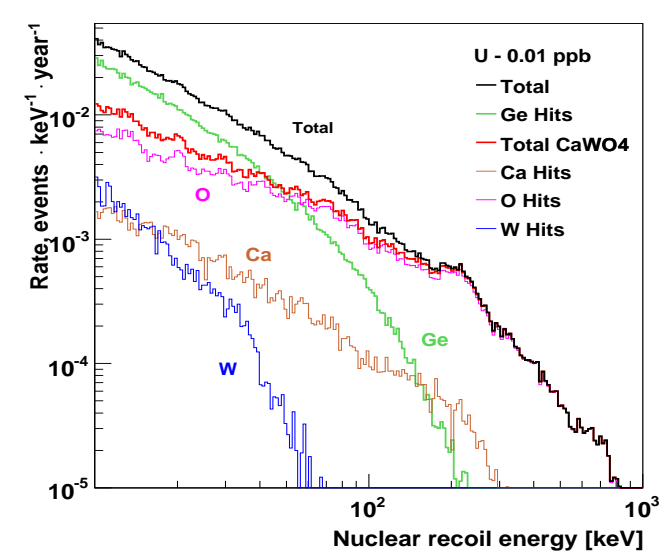

Figure 4: Nuclear recoil spectra from $0.01 \mathrm{ppb} \mathrm{U}$ in $\mathrm{Cu}$ of the cryostat for different types of recoiling nuclei $\left(253 \mathrm{~kg}\right.$ of $\mathrm{Ge}$ and $288 \mathrm{~kg}$ of $\mathrm{CaWO}_{4}$ were assumed as a target) [10].

in a cryostat and internal parts made out of 2.9 tonnes of copper. Figure 4 presents nuclear recoil spectra from $0.01 \mathrm{ppb} \mathrm{U}$ in $\mathrm{Cu}$ of the cryostat for different types of recoiling nuclei $(253 \mathrm{~kg}$ of $\mathrm{Ge}$ and $288 \mathrm{~kg}$ of $\mathrm{CaWO}_{4}$ were assumed as a target). Gamma-ray and neutron background from other detector materials and components has been studied for several running and planned experiments [11].

\section{Muon-induced neutrons}

At deep underground sites the background from radioactivity in rock dominates over cosmic- 
ray induced background. Neutrons produced by cosmic-ray muons should, however, be eliminated in experiments planning to reach high sensitivity to WIMPs or other rare events. Neutrons are produced by muons and muon-induced cascades not only in rock but also in all materials in and around a detector. The total neutron flux and, hence, the event rate in the detector are strongly affected by the composition of the rock and shielding, and the detector configuration. The background event rate due to muon-induced neutrons can be enhanced significantly in the presence of high-A target close to the detector. It can also be significantly reduced at energies below $10 \mathrm{MeV}$ by the presence of hydrogen in the shielding close to the detector. All these effects complicate simulations of the muon-induced neutron background. To make accurate predictions, full Monte Carlo of the experimental setup is needed.

The muon flux has been measured for most underground laboratories giving enough of information for testing Monte Carlo codes for muon simulations. Muons can be transported through large thickness of rock using general particle physics codes such as GEANT4 or FLUKA [12], or specially developed, simple, fast and widely used muon propagation codes such as MUSIC $[13,14]$. In most cases the muon transport through the rock is not needed and can be substituted with a sampling of muons according to the known muon energy spectrum and angular distribution at a particular depth. Such a sampling can be done with a code MUSUN [15, 14].

Two general-purpose codes GEANT4 and FLUKA have been used so far for production, transport and detection of muon-induced neutrons. The validation of the codes has been done through the comparison of them with each other $[16,17,19]$ and with available experimental data. Neutron yields and spectra calculated with GEANT4 and FLUKA agree with each other within 50\% for most materials $[16,17,19]$. The comparison between the Monte Carlo and experimental data is more tricky. Most experiments that measured muon-induced neutrons, did not present accurate Monte Carlo simulations which would include their set-ups together with production, transport and detection of all particles produced by muons. This makes the interpretation of experimental results difficult, since the experiments do not usually measure the neutron yields but the number of gamma-ray energy depositions due to neutron capture in a certain time window after the muon trigger and in a certain energy range. Conversion of this parameter into the neutron yield in a (possibly different) material is not straightforward and requires a full and accurate Monte Carlo simulations which were not possible to do at the time when most experiments were carried out. Nowadays such simulations are possible but not for the old data since it is difficult to find crucial details about old setups. Recent measurements of neutron yield for $\mathrm{CH}_{2}$ from KamLAND [20] agree quite well with simulation whereas the measured neutron yield in lead at Boulby [21] appears to be 2-3 times smaller than expected from GEANT4 (depending on the version of the code. Obviously the agreement between data and simulations for one material does not imply that the model is correct for other materials. Clearly more measurements accompanied by full Monte Carlo are needed to make sure that we know the muon-induced background with high degree of accuracy.

A few important points to note about simulations of muon-induced neutrons. Total neutron yields in light and heavy materials are very much different and the spectra of neutrons are different too [19]. The enhancement of the neutron flux In heavy materials occurs mainly at energies below $20 \mathrm{MeV}$, whereas the spectra above $20 \mathrm{MeV}$ are not much different (in shape and absolute fluxes) from those in light targets. Any extrapolation from one material to another one without accurate Monte Carlo may result in a significant error in the neutron flux. The mean neutron energies in 
different materials are: $65.3 \mathrm{MeV}\left(\mathrm{CH}_{2}\right), 23.4 \mathrm{MeV}(\mathrm{NaCl})$ and $8.8 \mathrm{MeV}(\mathrm{Pb})$ [19].

It is attractive to simplify simulations and save the CPU time by doing this in two stages and separating neutron production from neutron transport and detection. Such an approach may lead to a big error in the neutron event rate estimate due to the following reasons. Neutrons, and particularly fast neutrons, are not emitted isotropically with respect to the muon or any other parent particle direction. They are emitted preferentially along the parent particle path [22]. If the second stage of simulation starts with sampling neutrons (not muons) according to the pre-defined energy spectrum, the angular distribution of these neutrons is not known since the information about the direction, energy etc of the muon (or other particle that produced this neutron) was lost after the first step of simulation (neutron production by muons) was completed. Whatever assumption is made about the neutron angular distribution, it will not be accurate since muon angular distribution is not isotropic. Also, separating neutron transport and detection from neutron production means that an event caused by a neutron and accompanied by another energy deposition associated with a muon or other particles produced by the muon, will be seen as an event with nuclear recoil only, thus increasing the rate of background mimicking WIMP signal or any other rare event. Proper neutron multiplicity distribution should also be taken into account for each muon or muon-induced cascade. This is done automatically by following the development of the cascade and transporting all particles produced by a muon, but is difficult to achieve if neutron production is separated from neutron detection unless all particles are stored and transported at the 2nd stage of simulations.

The case of $250 \mathrm{~kg}$ xenon dark matter detector has been considered in Refs. [4, 16]. The spectra of energy depositions have been found to be very similar in GEANT4 and FLUKA [16]. Both codes predict similar rate of nuclear recoils and similar rejection factor for most events with nuclear recoils due to the presence of energy deposition from other particles in muon-induced cascades or muons themselves. In fact, only a few percent (5-8\%) of nuclear recoils in a large-scale xenon detector are 'pure' nuclear recoils without other energy depositions. Such coincidences will be missed if the simulation of production, propagation and detection are done for neutrons only but not for all other particles. So for correct simulations of neutron-induced effects it is very important to produce, transport and detect neutrons and all other particles associated with muons with a single, accurate Monte Carlo code such as GEANT4. avoiding parameterisations which may not be accurate enough.

The rate of single nuclear recoils from muon-induced neutrons in a large xenon detector is expected to be about $(9 \pm 3)$ events/tonne/year at 10-50 keV Xe recoil energies. Simulations included the $30 \mathrm{~cm}$ thick lead and $40 \mathrm{~g} / \mathrm{cm}^{2}$ hydrocarbon shielding. This is the rate of events not accompanied by any other energy deposition due to muons or muon-induced secondaries (electrons, photons, hadrons). With an active veto around the detector this rate can be reduced to below 1 event/tonne/year.

Similar studies have been recently carried out for the EURECA experiment planned to be located in the new hall of the Modane Underground Laboratory. In this case the two cryostats containing $\mathrm{Ge}$ and $\mathrm{CaWO}_{4}$ crystals were immersed into 2 water tanks with a $3 \mathrm{~m}$ thickness of water on all sides. A background rate of $1.6 \pm 0.5$ single nuclear recoils per year above $10 \mathrm{keV}$ was observed. If water is instrumented with PMTs and this Cherenkov veto system has a threshold of about $0.2 \mathrm{GeV}$, then all events observed in the detector will be rejected by anticoincidence with the veto resulting in an upper limit of 0.2 events per year above $10 \mathrm{keV}$ in a tonne-scale target. This 
will allow the experiment to reach the projected sensitivity to WIMP-nucleon spin-independent interactions below $10^{-10} \mathrm{pb}$.

\section{Acknowledgements}

I wish to thank many people who were involved in the ILIAS activities and other experimental and simulation efforts, the results of which are widely presented here. I am grateful to Drs. Matthew Robinson, Vito Tomasello and Mr. Edward Overton (University of Sheffield) for their work on the simulations for EURECA which formed the basis of the present paper.

\section{References}

[1] S. Agostinelli et al., Nucl. Instrum. and Meth. in Phys. Res. A, 506, 250 (2003); J. Allison et al. (Geant4 Collaboration), IEEE Trans. Nucl. Sci., 53, 270 (2006).

[2] V. I. Tretyak, The DECAY0 code, private communication.

[3] W. B. Wilson et al., SOURCES-4A, Technical Report LA-13639-MS, Los Alamos (1999).

[4] M. J. Carson et al., Astroparticle Phys., 21, 667 (2004).

[5] R. Lemrani et al., Nucl. Instrum. and Meth. in Phys. Res. A, 560, 454 (2006).

[6] V. Tomasello et al. Nucl. Instrum. and Meth. in Phys. Res. A, 595, 431 (2008).

[7] M. Herman et al., Nucl. Data Sheets, 1082655 (2007); http://www.nndc.bnl.gov/empire219.

[8] V. Tomasello, M. Robinson and V. A. Kudryavtsev, Astroparticle Physics, 34, 70 (2010).

[9] M. Selvi. Talk at the 8th Intern. Workshop on the Identification of Dark Matter (Montpellier, 2010).

[10] V. Tomasello. PhD Thesis, University of Sheffield (2010).

[11] See talks at the 8th Intern. Workshop on the Identification of Dark Matter (Montpellier, 2010).

[12] A. Ferrari, P. R. Sala, A. Fassò and J. Ranft, "FLUKA: A multi-particle transport code (Program version 2005)", CERN-2005-010, INFN/TC-05/11, SLAC-R-773; A. Fassò et al. Computing in High Energy and Nuclear Physics 2003 Conference (CHEP2003, La Jolla, USA, 2003), eConf C0303241.

[13] P. Antonioli et al., Astropart. Phys. 7, 357 (1997); V. Kudryavtsev et al., Phys. Lett. B 471, 251 (1999).

[14] V. A. Kudryavtsev, Computer Physics Communications, 180, 339 (2009).

[15] V. A. Kudryavtsev et al., Nucl. Instrum. \& Meth. in Phys. Res. A, 505, 688 (2003).

[16] H. M. Araújo et al., Nucl. Instrum. \& Meth. in Phys. Res. A, 545, 398 (2005).

[17] M. Bauer et al., Proc. 5th Intern. Workshop on the Identification of Dark Matter, ed. N. J. C. Spooner and V. A. Kudryavtsev (World Scientific, 2005), p. 494.

[18] M. Aglietta et al. (LVD Collaboration). Phys. Rev. D, 58, 092005 (1998).

[19] A. Lindote, H. M. Araújo, V. A. Kudryavtsev and M. Robinson, Astroparticle Physics, 31, 366 (2009).

[20] S. Abe et al. (KamLAND Collaboration), Phys. Rev. C, 81, 025807 (2010).

[21] H. M. Araújo et al., Astroparticle Physics, 29, 471 (2008).

[22] M. Horn. PhD Thesis, University of Karlsruhe (2007). 